\title{
FLUORESCENCE DIFFUSE OPTICAL IMAGE RECONSTRUCTION WITH A PRIORI INFORMATION
}

\author{
Lu Zhou, An Jin, Birsen Yazıcl \\ Rensselaer Polytechnic Institute \\ 110 8th St., Troy, NY 12180
}

\begin{abstract}
This paper addresses fluorescence diffuse optical tomography (FDOT) reconstruction problem with a priori information. We assume that approximate location of the fluorophore concentration is known from physiology of the disease and nature of the fluorophore injected. In addition, we assume the anatomical edge structures from an anatomical image modality partially coincident with the edges of the fluorophore concentration image. We formulate FDOT reconstruction in Bayesian framework and model a priori information of FDOT into a Gaussian Markov random field (GMRF) with several unknown parameters. We simultaneously estimate the optical image and the unknown a priori model parameters. Numerical simulations demonstrate that the a priori information could effectively improve the image reconstruction results.
\end{abstract}

Index Terms - fluorescence, optical tomography, image reconstruction

\section{INTRODUCTION}

Fluorescence diffuse optical tomography (FDOT) is a rapidly developing functional imaging modality that measures the 3D fluorophore activity inside biological tissue at cellular and molecular level [1]. FDOT requires 3D reconstruction of fluorophore concentration using boundary measurements obtained at the emission and excitation wavelength. However, the nonlinear and ill-posed nature of the FDOT inverse problem makes accurate reconstruction of fluorophore concentration a challenging problem.

Previous studies in the literature showed that incorporating structural a priori information from a high resolution imaging modality, such as magnetic resonance imaging (MRI) or X-ray computer tomography (CT), could improve the reconstruction of FDOT images. In [2,3], the structural a priori information from anatomical image is used to decomposes the fluorescence image domain into regions with

This work was supported by U.S. Army Medical Research Acquisition Activity under grant W81XWH-04-1-0559, Center for Subsurface Sensing and Imaging Systems, under the Engineering Research Centers Program of the National Science Foundation under Award Number EEC-9986821. similar anatomical properties. The reconstruction of fluorophore concentration is constrained spatially to particular segments. This can be considered a as 'hard-constraint' (as defined in [4]). However, it's always possible that the correlation between the a priori anatomical image and fluorophore concentration image may not be high. For example, the location of fluorophore concentration in the optical image may not have a corresponding counterpart in the anatomical image. In this case, imposing hard-constraint may lead to erroneous results. Thus the a priori information has to be incorporated as a 'soft-constraints' [5] by building a greater degree of uncertainty into the a priori model. A study by Davis et al [6] proposed a method to incorporate structural a priori information into a Laplacian-type matrix, where the a priori information is utilized as a soft-constraint to guide image reconstruction. Although Davis' study added some flexibility to deal with uncertainty in a priori information, the improvements in the accuracy of FDOT image reconstruction depends on the assumption of high structural-optical correlation.

In this work, we present an FDOT image reconstruction method where a priori information is utilized as a 'softconstraint' to guide image reconstruction, which can tackle with the case of low structural-optical correlation. We use two types of a priori information: (1) The localization information of the fluorophore concentration based on chemical properties of the fluorophore injected and the disease physiology. (2) The anatomical edge structure of the tissue. We build the a priori information into Gaussian Markov random field (GMRF) model with several unknown parameters. GMRF has been widely used for modeling spacial images, it captures both localization and edge information of fluorophore concentration. The unknown parameters of GMRF model are estimated iteratively from measurement data, which enables a flexible soft-constraint to guide image reconstruction. In simulation study, we show that when the structural-optical correlation is high, our method can significantly improve the reconstructed image. In addition, even if the correlation is low, our method can still get to good reconstruction results. We compare our method with to Laplacian-type regularization method [6], and show that when the a priori structural information deviates from the true optical image, the recon- 
struction results in our method are much less biased towards the prior as compared to the Laplacian-type regularization technique.

\section{FORWARD PROBLEM OF FDOT}

We model the forward problem of FDOT in frequency domain. In this work, we assume that the source is timeinvariant with zero modulation frequency, which is also known as continuous wave (CW) FDOT. Then the propagation of the excitation and emission light in a bounded domain $\Omega \subset \mathbb{R}^{3}$ is described by the following coupled diffusion equations:

$$
-\nabla \cdot D_{x}(\boldsymbol{r}) \nabla \phi_{x}\left(\boldsymbol{r}, \boldsymbol{r}_{i}\right)+\mu_{a x}(\boldsymbol{r}) \phi_{x}\left(\boldsymbol{r}, \boldsymbol{r}_{i}\right)=S_{i}(\boldsymbol{r}),
$$

$-\nabla \cdot D_{m}(\boldsymbol{r}) \nabla \phi_{m}\left(\boldsymbol{r}, \boldsymbol{r}_{i}\right)+\mu_{a m}(\boldsymbol{r}) \phi_{m}\left(r, \boldsymbol{r}_{i}\right)=\phi_{x}(\boldsymbol{r}) \eta \mu_{a x f}(\boldsymbol{r})$,

with the Robin boundary condition

$$
2 D_{x, m}(\boldsymbol{r}) \frac{\partial \phi_{x, m}\left(\boldsymbol{r}, \boldsymbol{r}_{i}\right)}{\partial \boldsymbol{n}}+\rho \phi_{x, m}\left(\boldsymbol{r}, \boldsymbol{r}_{i}\right)=0, \boldsymbol{r} \in \partial \Omega,
$$

where $\phi_{x, m}\left(\boldsymbol{r}, \boldsymbol{r}_{\boldsymbol{i}}\right)$ is the isotropic photon density of the excitation(emission) light at position $r$ due to the $i$ th point source $S_{i}(\boldsymbol{r})$ centered at $\boldsymbol{r}_{\boldsymbol{i}} . D_{x, m}(\boldsymbol{r})$ and $\mu_{a x, m}(\boldsymbol{r})$ are diffusion and absorption coefficients of the medium under excitation and emission wavelengths respectively. $c$ is the speed of light propagation in the medium. $\boldsymbol{n}$ is the direction perpendicular to the boundary, and $\rho$ is the boundary mismatch parameter due to the light reflection at the boundary. $\mu_{a x f}(\boldsymbol{r})$ is the absorption coefficient of the fluorophore at excitation wavelength, which is related to the fluorophore concentration and is the quantity to be reconstructed. $\eta$ is the known quantum yield.

In the case of weak fluorophore, it's reasonable to assume that in the NIR spectrum, the contribution of fluorophore absorption to the total absorption is negligible [7]. Thus we make the following simplifying assumptions:

$$
\begin{aligned}
\mu_{a x} & =\mu_{a x e}+\mu_{a x f} \approx \mu_{a x e} \\
\mu_{a m} & =\mu_{a m e}+\mu_{a m f} \approx \mu_{a m e}
\end{aligned}
$$

where the subscript $e$ denotes endogenous properties and $f$ denotes exogenous properties.

Let $\Gamma_{i, j}$ be the boundary measurement at the $j$ th detector due to the $i$ th source. Using (1)-(3), $\Gamma_{i, j}$ can be modeled as:

$$
\Gamma_{i, j}=\int_{\Omega} g^{*}\left(\boldsymbol{r}, \boldsymbol{r}_{j}\right) \phi_{x}\left(\boldsymbol{r}, \boldsymbol{r}_{i}\right) \eta \mu_{a x f} d \boldsymbol{r},
$$

where $g^{*}$ is the Green's function of adjoint problem associated with (2) [8]. If there are $N_{s}$ sources, $N_{d}$ detectors and the total number of measurements is $M=N_{s} \times N_{d}$, we can assemble $\Gamma_{i, j}$ into an $M \times 1$ vector as follows:

$$
\boldsymbol{\Gamma}=\left[\Gamma_{1,1}, \ldots, \Gamma_{1, N_{d}}, \ldots, \Gamma_{i, j}, \ldots, \Gamma_{N_{s}, N_{d}}\right]^{T},
$$

We discretize the domain into $N$ voxels and let $x_{i}=$ $\mu_{\text {axf }}\left(\boldsymbol{r}_{i}\right)$, where $\boldsymbol{r}_{i}$ is the center of $i$ th voxel.

$$
\boldsymbol{x}=\left[x_{1}, x_{2}, \ldots, x_{N}\right]^{T} .
$$

Discretizing (6) by the finite difference method, we get:

$$
\Gamma=A x,
$$

where $A: L^{2}(\Omega) \rightarrow \mathbb{R}^{M}$ is the vector-valued forward operator.

\section{BAYESIAN FDOT IMAGE RECONSTRUCTION WITH GMRF PRIOR MODEL}

\subsection{GMRF Prior Model}

We address the inverse problem within a Bayesian framework. We add zero-mean i.i.d white Gaussian noise $\varepsilon$ to the observation model in (7), and obtain:

$$
\boldsymbol{\Gamma}=A \boldsymbol{x}+\varepsilon,
$$

Thus the data likelihood is given by:

$$
p(\boldsymbol{\Gamma} \mid \boldsymbol{x})=\frac{1}{(2 \pi)^{M / 2} \sigma^{M}} \exp \left(-\frac{1}{2 \sigma_{n}^{2}}\|\boldsymbol{\Gamma}-A \boldsymbol{x}\|^{2}\right),
$$

where $\sigma^{2}$ is the variance of the addictive noise $\varepsilon$.

We model the prior density of $\boldsymbol{x}$ in GMRF as follows:

$$
\begin{array}{r}
p(\boldsymbol{x})=\frac{\exp \left\{U\left(\boldsymbol{x} \mid m_{k}, a_{k}, b_{k}^{1}, b_{k}^{2}, b_{k}^{3}\right)\right\}}{C\left(m_{k}, a_{k}, b_{k}^{1}, b_{k}^{2}, b_{k}^{3}\right)}, \\
U\left(\boldsymbol{x} \mid m_{k}, a_{k}, b_{k}^{1}, b_{k}^{2}, b_{k}^{3}\right)=-\sum_{k} a_{k}\left(x_{k}-m_{k}\right)^{2} \\
-\sum_{k}\left(\sum_{l=1}^{3} b_{k}^{l}\left(x_{k}^{l}\right)^{2}\right),
\end{array}
$$

where $C(\cdot)$ is the partition function, $U(\cdot)$ is known as the Gibbs energy, $x_{k}^{l}$ is the difference between $k$ th voxel $x_{k}$ and its neighboring pixel in the $l$ direction $(l=1,2,3)$ in $3 \mathrm{D}$ Cartesian coordinate system. $m_{k}, a_{k}, b_{k}^{1}, b_{k}^{2}, b_{k}^{3}$ are unknown parameters which vary by location $k$ corresponding to the center of the $k$ th voxel. $m_{k}$ is the mean value for $x_{k} . a_{k}$ penalizes the difference between $x_{k}$ and $m_{k} . a_{k}$ and $m_{k}$ model fluorophore localization information. $b_{k}^{l}$ penalizes the gradient in the $l$ direction. It models fluorophore edge structure across $l$ direction.

Using the a priori localization information, we decompose the image domain $\Omega$ into foreground $R_{f}$ and background $R_{b}$ regions. $R_{f}$ denotes the pixel locations where fluorophore is expected to accumulate, $R_{b}$ denotes the pixel locations where no fluorophore is expected. Similarly, for the edge information, we decompose $\Omega$ into region $R_{e}^{l}$ where fluorophore image is expected to have large gradient in the $l$ 
direction, i.e., an edge along the direction perpendicular to the $l$ direction, and $R_{n e}^{l}$ where no edge perpendicular to the $l$ direction is expected. Next, we assume that $m_{k}, a_{k}, b_{k}^{l}$ are unknown piece-wise constant functions on the corresponding regions:

$$
\begin{aligned}
& m_{k}=\left\{\begin{array}{cc}
m_{f} & \boldsymbol{r}_{k} \in R_{f} \\
m_{b} & \boldsymbol{r}_{k} \in R_{b}
\end{array} \quad, \quad a_{k}=\left\{\begin{array}{cc}
a_{f} & \boldsymbol{r}_{k} \in R_{f} \\
a_{b} & \boldsymbol{r}_{k} \in R_{b}
\end{array},\right.\right. \\
& b_{k}^{l}=\left\{\begin{array}{ll}
b_{e}^{l} & \boldsymbol{r}_{k} \in R_{e}^{l} \\
b_{b}^{l} & \boldsymbol{r}_{k} \in R_{n e}^{l}
\end{array} \quad, l=1,2,3 .\right.
\end{aligned}
$$

Let $\boldsymbol{\theta}=\left[m_{f}, m_{b}, a_{f}, a_{b}, b_{e}^{1}, b_{b}^{1}, b_{e}^{2}, b_{b}^{2}, b_{e}^{3}, b_{b}^{3}\right]^{T}$ denote the unknown parameter vector of the GMRF prior model. The MAP estimate of $\boldsymbol{x}$ can be obtained from the posterior density function of $\boldsymbol{x}$ as a function of $\boldsymbol{\theta}$. Thus,

$$
\hat{\boldsymbol{x}}=\arg \max _{\boldsymbol{x}} p(\boldsymbol{\Gamma} \mid \boldsymbol{x}) p(\boldsymbol{x})
$$

\subsection{Parameters estimation}

In this section, we estimate the unknown parameters vector $\boldsymbol{\theta}$ from measurements as follows:

$$
\boldsymbol{\theta}=\arg \max _{\boldsymbol{\theta}} J(\boldsymbol{\theta}) \triangleq \arg \max _{\boldsymbol{\theta}} E_{\boldsymbol{x}}[\log p(\boldsymbol{\Gamma}, \boldsymbol{x}) \mid \boldsymbol{\Gamma}, \boldsymbol{\theta}] .
$$

The optimal parameters for (13) can be found iteratively by the EM algorithm [9]:

$$
\begin{aligned}
& \left(\boldsymbol{\theta}^{(q+1)}\right)=\arg \max _{\boldsymbol{\theta}} E_{\boldsymbol{x}}\left[\log p(\boldsymbol{\Gamma}, \boldsymbol{x}) \mid \boldsymbol{\Gamma}, \boldsymbol{\theta}^{(q)}\right] \\
& =\arg \max _{\boldsymbol{\theta}} E_{\boldsymbol{x}}\left[-\frac{\|\boldsymbol{\Gamma}-A \boldsymbol{x}\|^{2}}{2 \sigma^{2}}\right. \\
& \left.-\log C(\boldsymbol{\theta})+U(\boldsymbol{x} \mid \boldsymbol{\theta}) \mid \boldsymbol{\Gamma}, \boldsymbol{\theta}^{(q)}\right] \\
& \triangleq \quad \arg \max _{\boldsymbol{\theta}} F\left(\boldsymbol{\theta} \mid \boldsymbol{\Gamma}, \boldsymbol{\theta}^{(q)}\right) \text {, }
\end{aligned}
$$

where $\boldsymbol{\theta}^{(q)}$ is the parameters vector estimated at the $q$ th iteration of the EM algorithm. The pseudo code is given in Algorithm 1.

\section{SIMULATION RESULTS}

In this section, we simulated the FDOT image reconstruction using a 2-D phantom shown in Figure 1(a). The reconstruction area is a $6 \mathrm{~cm} \times 6 \mathrm{~cm}$ square. The origin of the coordinate system is located at the center of this phantom. The optical properties for the phantom are: $\mu_{a}=0.05 \mathrm{~cm}^{-1}$ and $\mu_{s}^{\prime}=8 \mathrm{~cm}^{-1}\left(D=1 / 3\left(\mu_{a}+\mu_{s}^{\prime}\right)\right)$. Within the phantom, $c=2 \times 10^{10} \mathrm{~cm}$, and on the boundary of phantom, $\rho=0.5$. The fluorophore with $\mu_{a f}=0.005 \mathrm{~cm}^{-1}$ and $\eta=0.05$ is in a $1.2 \times 1.2 \mathrm{~cm}$ square-shaped region, and the center of this region is located at $(-1.20,0)$. We place 20 sources (black triangles) and 25 detectors (hollow diamonds) on the boundary as shown in Figure 1(a). We add 10\% i.i.d. Gaussian noise into the boundary data.

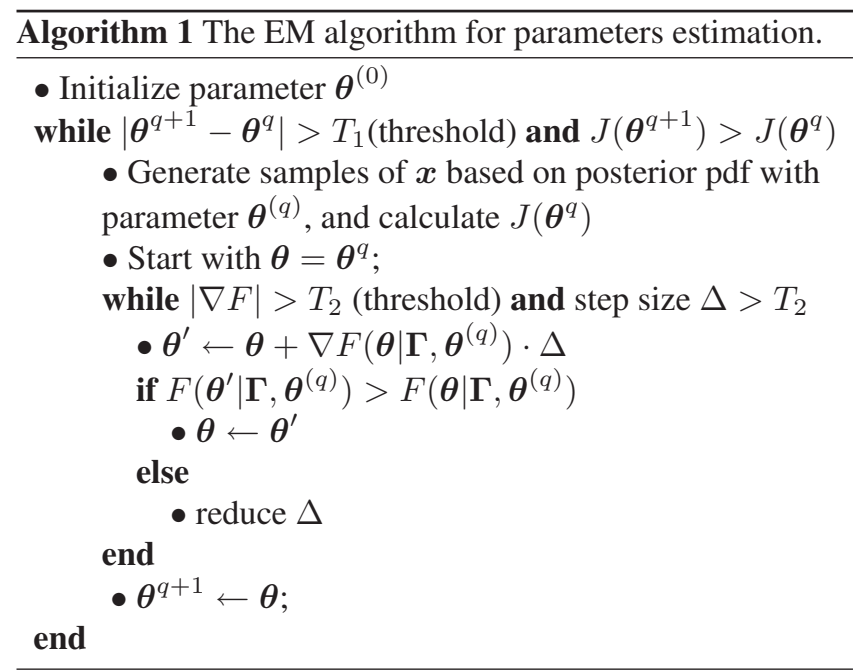

The a priori information used in the simulation is presented in Figure $1(\mathrm{~b})$. The $1.2 \mathrm{~cm} \times 1.2 \mathrm{~cm}$ shaded area indicates the a priori foreground $R_{f}$, and the blank area indicates the a priori background $R_{b} . R_{e}^{l}, l=1,2$, corresponds to the edges of $R_{f}$, and $R_{n e}^{l}, l=1,2$, is the the region where no edge is expected across $l$ direction. As we move $R_{f}$ (shown in Figure 1(b)), the a priori localization and edge information deviates from the true corresponding information in the fluorophore concentration image.

Figures 2(a)- 2(c) show the reconstruction results when the a priori foreground region is centered at $(-1.2,0)$, $(-0.3,0)$ and $(0.3,0)$, respectively. For comparison, we reconstructed the fluorophore concentration using the Laplacian type regularization [6], which is shown in Figures 2(d)- 2(f), respectively. We observe that in Figure 2(a) and Figure 2(d), where the structural-optical correlation is high, both methods obtain good reconstruction results. However, when the a priori information deviates from the true fluorophore concentration image, the images reconstructed by our approach (shown in Figure 2(b) and Figure 2(c)) are less biased towards the prior as compared to the ones reconstructed by the Laplacian-type regularization approach as shown in Figure 2(e) and Figure 2(f).

To evaluate the results quantitatively, we calculate the contrast-to-background noise ratio $(\mathrm{CBNR})$ and the contrastto-object noise ratio (CONR) of the reconstructed image and summarized them in Table1. The quantitative result is consistent with the visual result presented in Figure 2.

\section{CONCLUSION}

In this work, we formulated a priori information of FDOT by a GMRF model with unknown parameters. These parameters capture a priori information on the location and the gradient of fluorophore concentration. We estimated the unknown $a$ priori model parameters from measurements using the EM 


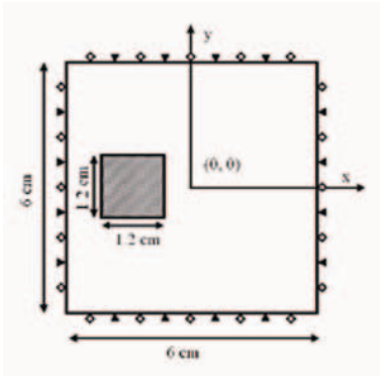

(a) The simulation setup.

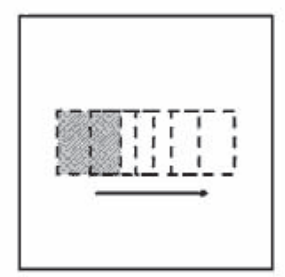

(b) The prior structural image.

Fig. 1. The simulation setup and prior structural image.

Table 1. CBNR and CONR of the reconstruction images in simulation.

\begin{tabular}{|c|c|c|c|c|}
\hline \multirow{2}{*}{$R_{f}$ center } & \multicolumn{2}{|c|}{ CBNR } & \multicolumn{2}{c|}{ CONR } \\
\cline { 2 - 5 } & Proposed & Lapalcian & Proposed & Laplacian \\
\hline$(-1.2,0.0)$ & 26.46 & 29.00 & 10.35 & 27.70 \\
$(-0.3,0.0)$ & 7.177 & 4.478 & 4.744 & 3.513 \\
$(0.3,0.0)$ & 7.639 & 4.735 & 3.806 & 2.352 \\
\hline
\end{tabular}

algorithm. Numerical results show that GMRF model is robust with respect to imperfect a priori information. The future work will include applying this algorithm to real phantom or clinical data.

\section{REFERENCES}

[1] V. Ntziachristos, "Fluorescence molecular imaging," Annu. Rev. Biomed. Eng, vol. 8, pp. 1-33, 2006.

[2] W. Cong, G. Wang, D. Kumar, Y. Liu, M. Jiang, L. Wang, E. Hoffman, G. McLennan, P. McCray, J. Zabner, and A.Cong, "Practical reconstruction method for bioluminescence tomography," Optics Express, vol. 13(18), pp. 6756-6771, 2003.

[3] Y. Lin, H. Gao, O. Nalcioglu, and G. Gulsen, "Fluo-

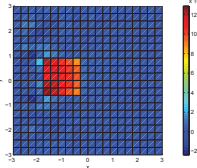

(a)

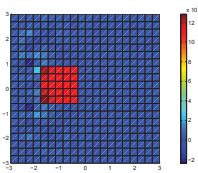

(d)

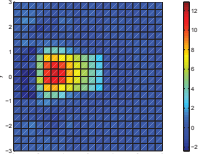

(b)

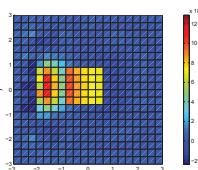

(e)

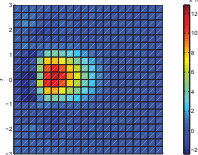

(c)

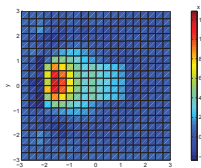

(f)
Fig. 2. (a), (b) and (c) are images reconstructed using the proposed algorithm with expected foreground centered at $(-1.2,0),(-0.3,0)$ and $(0.3,0)$ respectively. (d), (e) and (f) are images reconstructed using the Laplacian type regularization with expected foreground centered at $(-1.2,0)$, $(-0.3,0)$ and $(0.3,0)$ respectively.

rescence diffuse optical tomography with functional and anatomical a priori information: feasibility study," Phys. Med. Biol, vol. 52, pp. 5569-5585, 2007.

[4] H. Dehghani, B.W. Pogue, J. Shudong, B. Brooksby, and K.D. Paulsen, "Three-dimensional optical tomography: resolution in small-object imaging," Appl. Opt., vol. 42, pp. 3117-3126, 2003.

[5] P.K. Yalavarthy, B.W. Pogue, H. Dehghani, C.M. Carpenter, S. Jiang, and K.D. Paulsen, "Structural information within regularization matrices improves near infrared diffuse optical tomography," Optics Express, vol. 15, pp. 8043-8058, 2007.

[6] S.C. Davis, H. Dehghani, J. Wang, S. Jiang, B.W. Pogue, and K.D. Paulsen, "Image-guided diffuse optical fluorescence tomography implemented with laplacian-type regularization," Optics Express, vol. 15, pp. 4066-4082, 2007.

[7] V. Ntziachristos and R. Weissleder, "Experimental threedimensional fluorescence reconstruction of diffuse media by use of a normalized born approximation," Optics Letters, vol. 26(12), pp. 893-895, 2001.

[8] S.R. Arridge, "Optical tomography in medical imaging," Inverse Problem, vol. 15, pp. R41-R93, 1999.

[9] C.A. Bouman S. Saquib and K. Sauer, "Ml parameter estimation for markov random fields with applications to bayesian tomography," IEEE Transactions on image processing, vol. 7, pp. 1029-1044, 1998. 